\title{
Carnosine—a natural bioactive dipeptide: bioaccessibility, bioavailability and health benefits
}

\author{
Lujuan Xinga,b, MacKenzie E. Chee ${ }^{\mathrm{b}}$, Hua Zhang ${ }^{\mathrm{b}}$, \\ Wangang Zhang a and Yoshinori Mine ${ }^{\mathrm{b}^{*}}$
}

\begin{abstract}
aLaboratory of Meat Processing and Quality Control of EDU, College of Food Science and Technology, Nanjing Agricultural University, Nanjing, Jiangsu 210095, China

${ }^{b}$ Department of Food Science, University of Guelph, Guelph, Ontario N1G 2W1, Canada

${ }^{*}$ Corresponding author: Yoshinori Mine, Department of Food Science, University of Guelph, Guelph, Ontario, Canada N1G2W1. Tel: 1-519-824-4120 Ext 52901; Fax: 1-519-824-6631; E-mail: ymine@uoguelph.ca
\end{abstract}

DOI: $10.31665 / J F B .2019 .5174$

Received: January 15, 2019; Revised received \& accepted: March 15, 2019

AbbreviationsAD, Alzheimer's disease; hPHT1, human peptide/histidine transporter 1; Trolox, vitamin E derivative; MDA, malondialdehyde; AGEs, advanced glycation end products; HCD, histidine-containing dipeptides; NAC, N-alpha-acetyl-carnosine; GSH, Glutathione; $\mathrm{SOD}$, Superoxide dismutase; ROS, Reactive Oxygen Species; THC, trihexosylceramide; PC, phosphatidylcholine; $\mathrm{H}_{2} \mathrm{O}_{2}$, hydrogen peroxide; TNF- $\alpha$, tumour necrosis factor.

Citation: Xing, L., Chee, M.K.E., Zhang, H., Zhang, W., and Mine, Y. (2019). Carnosine - a natural bioactive dipeptide: bioaccessibility, bioavailability and health benefits. J. Food Bioact. 5: 8-17.

\begin{abstract}
Carnosine is a natural dipeptide synthesized by both vertebrate and invertebrate organisms and has functional properties that are specific to muscle and excitable tissues. Recent in vivo and in vitro studies have shown that carnosine presents metal chelating and antioxidant activities and has the ability to inhibit protein carbonylation and glycoxidation. This review describes the health benefits of carnosine in relation to its bioaccessibility, bioavailability and biochemical properties as well as providing the current state-of-the-art knowledge on the potential use of carnosine as a nutraceutical. The therapeutic potential of carnosine has also been investigated by a number of preclinical and clinical studies for diseases such as diabetes and its associated complications, as well as fatigue, ageing, and some neurological disorders. Altogether the current literature provides supportive evidence on the use of carnosine as a natural dietary supplement with significant health boosting efficiency and without any side effects.
\end{abstract}

Keywords: Carnosine; Bio-acitve peptides; Carnosinase; Anti-oxidant; Anti-glycation.

\section{Introduction}

Carnosine ( $\beta$-alanyl-L-histidine) is a naturally occurring cytoplasmic dipeptide widely distributed in both vertebrate and invertebrate organisms, and highly concentrated in tissues such as brain, eye lens and skeletal muscles. Carnosine has a number of derivatives (Figure 1) with the most common variants being anserine and ophidine, both comprising a methylated imidazole ring of Lhistidine (Boldyrev et al., 2013). Since its first isolation from meat extract, a number of in vitro and in vivo studies have investigated the physiological and biochemical properties of carnosine. The biological function of carnosine as a metal ion chelator, as well as its physiological $\mathrm{pH}$ buffering, free radical scavenging, anti-glycation and anti-lipid peroxidation activities, have now been well established (Boldyrev et al., 2013). Recognized as a potent antioxidant molecule, carnosine is generally associated with pleiotropic pharmacological effect, such as anti-oxidation, anti-inflammation, antiageing, neuroprotection e.g., prevention of Alzheimer's disease (AD), diabetes regulation as well as therapeutic effects on ocular disorders. Recently, carnosine was shown to inhibit oxidative nonenzymatic modifications as well as reverse advanced glycoxida- 


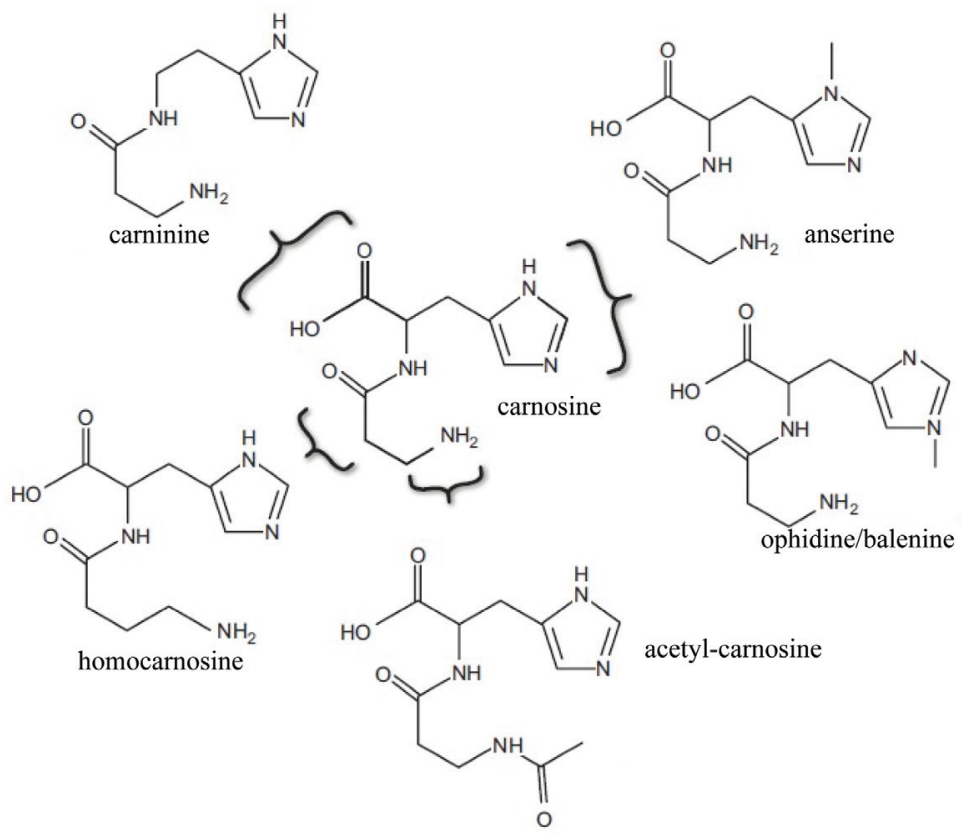

Figure 1. Chemical structure of carnosine ( $\beta$-alanine-L-histidine) and its naturally occurring derivatives (adapted from Boldyrev et al., 2013).

tion and lipid oxidation end product. These results have supported its clinical use for the prevention of chronic diseases such as type 2 diabetes, diabetic nephropathy, and cardiovascular neurodegenerative diseases (Baye et al., 2017). A number of investigations have described the functionality of carnosine and ascertained its health benefits in humans. The value of carnosine as a nutritional supplement/nutraceutical remains unknown to the general public. Hence this review presents the most recent advances on the bioaccessibility, bioavailability, and biological activities of carnosine.

\section{Chemical characterization}

Carnosine is a water-soluble cytoplasmic dipeptide comprised of $\beta$-alanine and L-histidine. The imidazole ring of L-histidine confers the dipeptide its biological activity while $\beta$-alanine has a regulatory functions on its rate of synthesis (Boldyrev et al., 2013). Carnosine is synthesized by carnosine synthetase and hydrolyzed by carnosinase, which are both primarily expressed in the muscle tissue and in some areas of the brain (di Pierro et al., 2011). The rate of the enzymatic condensation reaction is limited by the availability of $\beta$-alanine (Derave et al., 2010). The hydrolysis of carnosine occurs predominantly in the serum where carnosinase activity is higher compared to other tissues. (Lenney et al., 1985). There are two forms of carnosinase: $\mathrm{CN} 1$, which is present in the serum and $\mathrm{CN} 2$, which is expressed in tissues. CN2 accounts for a significantly smaller fraction of the dipeptide's breakdown in humans (Derave et al., 2010). In contrast, carnosine is found in all excitable tissues of the human body, including brain, myocardium, and skeletal muscle (Kyriazis, 2010), however, 99\% of the body's carnosine is present in the muscle (Derave et al., 2010). In mammals, only skeletal muscle and olfactory bulb present millimolar concentration range of carnosine while brain regions, other tissues and body fluids have 10- to 1,000- fold lower concentration (Boldyrev et al., 2013). The acid dissociation constant, pKa value (6.83) of the imidazole ring of histidine confers the dipeptide its
$\mathrm{pH}$ buffering ability in muscle cells, which maintains $\mathrm{pH}$ and promotes exercise capacity by counteracting the enhanced production of lactic acid during prolonged physical activity ( Park et al., 2005; di Pierro et al., 2011). Contrastingly, free histidine has a pKa value of 5.83 and is a poor physiological buffer compared to carnosine (Hill et al., 2007).

\section{Isolation of carnosine from meat products}

Generally, carnosine is highly abundant in the muscle of animals, however its content may be affected by the type of breed, gender, breeding activity and age (Crush, 1970). Carnosine content in pork, beef, chicken, and seafood is summarized in Table 1. In all six types of commercial chicken, carnosine content ranged from 0.66 to $1.83 \mathrm{mg} / \mathrm{g}$, interestingly, carnosine content was 7-fold higher in the chicken breast compared to thigh muscles (Jung et al., 2013). Content was quantified at 35 and $104 \mathrm{mg} / 100 \mathrm{~g}$ in female chicken thigh and breast muscle, respectively; as opposed to 30 and $71 \mathrm{mg} / 100 \mathrm{~g}$ in males (Peiretti and Meineri, 2015). An opposing trend was described in the duck muscle, with female ducks showing lower levels of carnosine compared to males, suggesting that gender differences vary between species (Lee et al., 2015). Anserine concentrations were found to be higher than carnosine in the breast and thigh meat extract of chicken as well as turkey (Huang and Kuo, 2000; Peiretti et al., 2012). In beef extract, carnosine content was quantified as $3.79 \mathrm{mg} / \mathrm{g}$ in raw meat (Chan et al., 1993) while in cured beef products, higher concentrations ranging from 5.8 to $7.1 \mathrm{mg} / \mathrm{g}$, were found (Marcolini et al., 2015). A higher concentration of carnosine in cured beef product would be related with the loss of water during the processing of meat. In the study of Purchas et al. (2004), the content of carnosine in uncooked beef muscle was $4.1 \mathrm{mg} / \mathrm{g}$, which was decreased to be $3.3 \mathrm{mg} / \mathrm{g}$ after the cooking process. The bioactive molecule carnosine was watersoluble cytoplasmic dipeptide, thus during the cooking process, the loss of carnosine was related with the generation of cooking 
Table 1. Carnosine contents in raw meat extract obtained from different kind of species

\begin{tabular}{|c|c|c|}
\hline Type of extract & Carnosine concentration (mg/g) & Reference \\
\hline Beef & $5.8 \sim 7.12$ & Marcolini et al., 2015 \\
\hline Pork & $0.13 \sim 4.19$ & Mora et al., 2008 \\
\hline Horse & $1.22 \sim 4.07$ & Abe, 2000 \\
\hline Lamb & 7.06 & Jones et al., 2011 \\
\hline Rabbit & $3.6 \sim 4.6$ & Peiretti and Meineri, 2015 \\
\hline Chicken & $0.66 \sim 1.83$ & Jung et al., 2013 \\
\hline Turkey & $0.86 \sim 7.9$ & Gil-Agustí et al., 2008, Peiretti et al., 2012 \\
\hline Duck & $0.14 \sim 0.16$ & Lee et al., 2015 \\
\hline Lobster & 0.1 & Kantha et al., 2000 \\
\hline Prawn & $9.25 \sim 11.6$ & Preedy, 2015 \\
\hline Tuna & 5.29 & Jones et al., 2011 \\
\hline Mackerel & 7.78 & Jones et al., 2011 \\
\hline Rainbow Trout & 0.11 & Jones et al., 2011 \\
\hline Bonito (katsuo) & $5.0 \sim 7.9$ & Kantha et al.,2000 \\
\hline Conger eel (hamo) & 0.2 & Kantha et al., 2000 \\
\hline
\end{tabular}

juices. In pork, carnosine content was compared between seven types of muscle with concentrations ranging from 0.13 to 4.19 $\mathrm{mg} / \mathrm{g}$. A higher carnosine content was found to be associated with glycolytic muscles e.g., the semimembranosus, the biceps femoris, the gluteus maximus and the longissimus dorsi (Mora et al., 2008). According to Suzuki, et al. (2002), this might be explained by the high buffering capacity of carnosine which is a vital feature for glycolytic muscles, where anaerobic metabolism results in the accumulation of lactic acid. Seafood and prawns also present high content of carnosine ranging within $9.25-11.6 \mathrm{mg} / \mathrm{g}$, while fish has lower content ranging from 0.11 to $7.78 \mathrm{mg} / \mathrm{g}$, e.g., tuna, mackerel and rainbow trout (Jones et al., 2011; Preedy, 2015), whether it is boiling or broiling, the cooking process cause up to $50 \%$ loss of carnosine in beef and turkey meat, while the degradation of carnosine is lessened during microwave cooking (Peiretti and Meineri, 2015). The use of microwave cooking would thus help reduce the loss of carnosine and imidazole-related compounds.

\section{Bioaccessibility}

As a bioactive peptide widely found in meat and meat products, carnosine intake mainly relies on the absorption of meat protein through the gastrointestinal tract. It was determined that, upon digestion, the amount of dietary carnosine absorbed through the intestine was strongly associated with its original concentration in the food product, as well as with the amount that could be released from the food matrix. Contributing factors, such as $\mathrm{pH}$ in the intestine, affect the concentration of carnosine released from food e.g., beef, as it was shown that an in vitro digestive treatment at $\mathrm{pH} 2$ led to higher release of carnosine compared to $\mathrm{pH} 7$ (Marcolini et al., 2015). Importantly, the chewing and subsequent acidification occurring during the digestion process were shown to achieve more than $90 \%$ release of carnosine from cured beef products. Upon release, the absorption of carnosine is mainly mediated via proton-coupled peptide transporters in the small intestine (Son et al., 2004) as represented in Figure 2, while the paracellular transport of intact carnosine was ruled out by earlier authors. Son et al. (2004) also examined the transport of carnosine in a Caco-2 epithelium cell model and found that the dipeptide's transport was mediated by the proton-dependent peptide transporter PepT1. The authors showed an enhanced PepT1-mediated uptake of carnosine that occurred when the $\mathrm{pH}$ of the transport solution decreased from 7.4 to 6.0. Competitive uptake was observed when other dipeptides were present in the transport solution, while inclusion of amino acids did not affect the uptake of carnosine. PepT1 transporter is expressed in the small intestine, renal proximal tubule, and liver (Bhardwaj et al., 2006). Ferraris, et al. (1988) found that carnosine uptake was at its highest in the jejunum of mice. Upon PepT1mediated transport across the brush-border membrane, carnosine is faced with two fates: (1) hydrolysis by carnosinase within the enterocyte or (2) transport across the basolateral membrane into the blood as an intact dipeptide (Boldyrev et al., 2013). The mechanism underlying the basolateral transport of carnosine enterocytes is more obscure than its apical counterpart, and warrants further investigations.

Derave et al. (2010) speculated that the release of carnosine from muscle cells may be mediated by PepT2, since PepT2-knockout mice were found to have reduced muscle carnosine concentrations. It was also hypothesized that the reduction in muscle carnosine levels during exercise-induced acidosis may be mediated by the proton-dependent PepT2 transporter. PepT2 is known to be in an integral transporter in the reabsorption of carnosine in the renal tubules (Boldyrev et al., 2013). The PepT2-mediated apical transport of carnosine across renal epithelial cells occurred at 15 times higher rate compared to its basolateral transport (Jappar et al., 2009). It was hence hypothesized that the efflux of carnosine from renal epithelial cells was regulated by a facilitative carrier, which would limit its transepithelial transport. Carnosine accumulates to some degree within the renal epithelial cell and undergoes degradation by $\mathrm{CN} 2$ leading to the release of histidine and $\beta$-alanine, which then moves across the basolateral membrane via amino acid transporters. Finally, Histidine and $\beta$-alanine are subsequently reassembled in the tissue via the enzymatic activity of carnosine 


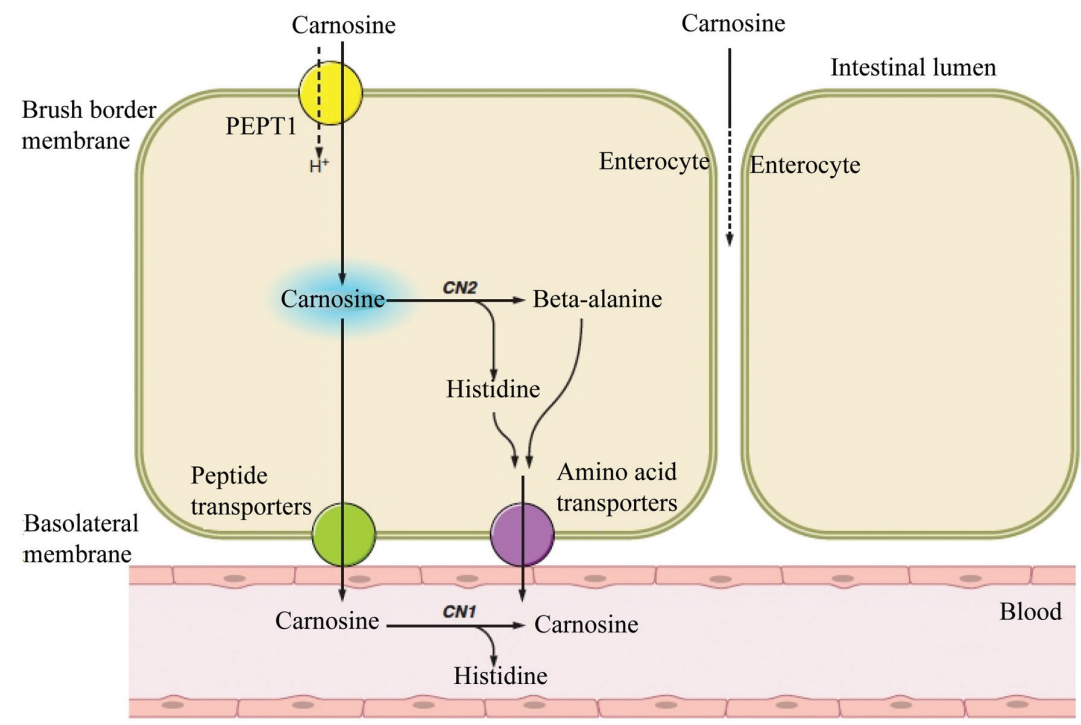

Figure 2. Proposed mechanisms of carnosine uptake by enterocytes. Carnosine uptake is mediated by the proton-dependent peptide transporter PepT1. It is subsequently hydrolyzed within the enterocyte by tissue carnosinase (CN2) or transported intact into the blood where it is hydrolyzed by serum carnosinase (CN1) into its substituents $\beta$-alanine and L-histidine. (adapted from Boldyrev et al., 2013).

synthetase, as shown in Figure 2. Another transport system was described by Bhardwaj et al., (2006), whereby the proton-dependent uptake of intact carnosine was mediated by the human peptide/ histidine transporter 1 (hPHT1) in a transfected monkey kidney COS-7 cell system. Carnosine uptake via hPHT1 was observed to increase as $\mathrm{pH}$ decreased, and transport was determined to be optimal at $\mathrm{pH}$ 5.0. Through mRNA and protein analysis of gut sections and protein lysates obtained from the human gastrointestinal tract, PHT1 was found to be mainly expressed in the epithelium of the small intestine. Knowledge on the transport of carnosine via hPHT1 remains scarce and requires further investigations.

An additional gap in the understanding of carnosine bioaccessibility resides in the translation of results obtained in animal models (i.e., hamsters, mice, horses, pigs) to human applications. Findings resulting from these animal models should be cautiously interpreted, as the CN1 levels in humans are significantly higher than those found in animals e.g., misinterpretation of the levels of carnosine found in serum (Boldyrev et al., 2013; Derave et al., 2010).

\section{Bioavailability}

While carnosine was reported as being a bioavailable dietary peptide capable of reaching the blood in an intact form via absorption through the gastrointestinal tract, its oral bioavailability remains limited (di Pierro et al., 2011). In the transcellular absorption, the cellular uptake of carnosine was mediated by PEPT2 at the apical membrane, and the efflux from basolateral membrane into blood was very low. The limited bioavailability of carnosine is significantly associated with its poor basolateral transport. Compare with carnosine, the uptake of $\beta$-alanine was easier to be absorbed by the intestinal cells. It was documented that supplementation with the rate-limiting precursor $\beta$-alanine, rather than carnosine itself, can produce up to $80 \%$ increase in carnosine levels in the skeletal muscle tissue (Derave et al., 2010). A galenic formulation was developed that contained equal amounts of pure carnosine and pure $\beta$-alanine after 8- hour oral ingestion. Finally, oral ingestion of a dietary supplement containing carnosine was found not to increase carnosine levels in muscle more than a supplement containing equimolar amount of $\beta$-alanine alone (Derave et al., 2010). In support of its bioaccessibility, a study in which mini-pigs were fed various types of meat showed that the hydrolytic degradation of carnosine in the gut lumen did not occur fast enough to prevent absorption of the dipeptide across the gastrointestinal tract (Bauchart et al., 2007). Previous authors developed complexes composed of carnosine and Trolox, a vitamin E analog, to inhibit degradation by human serum carnosinase. They found that the carnosine derivatives performed better in reducing oxidative stress compared to carnosine or Trolox individually, but also showed more resistance to serum carnosinase degradation (Stvolinsky et al., 2010).

In a human cohort study, Park et al. (2005) examined the effects of beef consumption on serum carnosine levels at given time intervals. Subjects were asked to abstain from carnosine-containing food for 24 hours prior to the study to ensure a consistent baseline level of serum carnosine, which was confirmed by blood collection. Subjects then consumed $200 \mathrm{~g}$ of cooked ground beef containing a total of $268 \mathrm{mg}$ carnosine and subsequently had their blood collected at regular intervals. As represented in Figure 3, carnosine was detected in the blood as early as 15 minutes after ingestion. Carnosine concentrations in serum peaked at 3.5 hours post-ingestion and went below detection levels in blood after 5.5 hours. In a study by Marcolini et al. (2015), the gap between total carnosine content and its free diffusible fraction was significant in bresaola products. The authors concluded that carnosine was easily released from the food matrix upon digestion within the gastrointestinal tract hence available for optimal absorption.

Hill et al. (2007) examined the effects of $\beta$-alanine supplementation on carnosine concentrations found in the muscle. Subjects were given increasing doses of $\beta$-alanine every day for 4 vs. 10 weeks. The orally ingested dose of $\beta$-alanine started at $4.0 \mathrm{~g}$ per day, divided into 8 separate supplement ingestion. The dose of $\beta$-alanine was gradually increased by $0.6-0.8 \mathrm{~g}$ on a weekly basis until week 4 when the dose reached $6.4 \mathrm{~g}$ per day. Carnosine concentrations were measured in vastus lateralis biopsies and were found to increase by $58.8 \%$ and $80.1 \%$ in the 4 th and 10 week treatment groups, respectively (Hill et al., 2007). As mentioned 


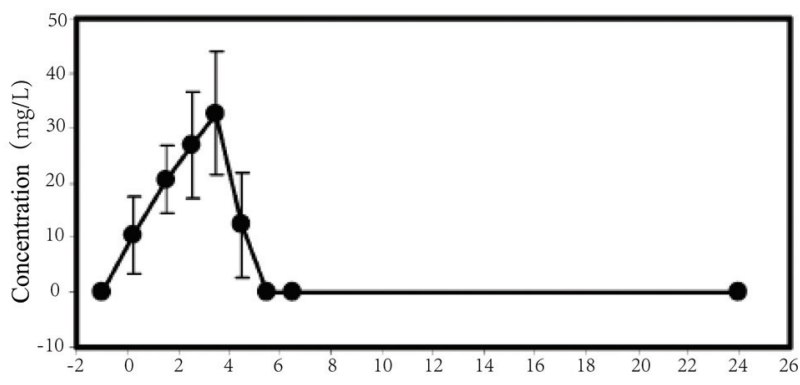

Figure 3. Serum concentration of carnosine in subjects post-consumption of $\mathbf{2 0 0 ~ m g ~ c o o k e d ~ g r o u n d ~ b e e f ~ c o n t a i n i n g ~} 134 \mathrm{mg} / \mathrm{g}$ tissue (cooked) of carnosine (adapted from Park et al., 2005).

previously, supplementation with carnosine or $\beta$-alanine both resulted in an increased amount of carnosine in muscle, whereas $\beta$-alanine alone elicited a greater increase than carsosine (Derave et al., 2010).

In the human muscle, the concentrations of carnosine classically reach up to $20 \mathrm{mM} / \mathrm{kg}$ (dry weight of muscle) and ingestion of supplement made of carnosine, $\beta$-alanine or meat products would all promote an increasing effect (Derave, Everaert, Beeckman, and Baguet, 2010). The main differences between the ingestion of carnosine from food matrix vs. supplement e.g., capsule, would be in its bioaccessibility and bioavailability. Indeed, the release and absorption rate of carnosine from meat products would be proportional to its original concentration in the food product and would be dependent on the gastrointestinal environment, while the absorption rate of carnosine upon ingestion of capsule supplements would be equal to the dose indicated on the label (Marcolini et al., 2015). So far there have been no reports indicating differences in the biological activity of carnosine depending on the source or nature of the food matrix ingested. Thus, the direct supplementation of carnosine capsule or uptake the food rich in carnosine would all possess the benefit for the health of body.

\section{Metabolism}

In the diet, the supplement of carnosine was mainly reliant on the ingestion of meat and therefore the vegetarian diet would lack carnosine. The provision of carnosine through the diet is mainly relliant on the ingestion of meat; hence a vegetarian diet would result in carnosine deficiency. Once in the serum or the tissue, carnosine is degraded by carnosinase. In humans, CN2 is almost completely absent from the skeletal muscle tissue; hence $\mathrm{CN} 1$ is responsible for the majority of its degradation (Derave et al., 2010). Carnosine was shown to be resistant to breakdown by peptidases (Boldyrev et al., 2013; Hipkiss et al., 1995), while a non-enzymatic breakdown of carnosine does not exist (Derave et al., 2010). Therefore, the very specific degradation of carnosine suggests that its metabolism is tightly regulated. The rate-limiting precursor $\beta$-alanine results from the hepatic breakdown of uracil and thymidine as well as from the degradation of dietary dipeptides derived from meat sources (Derave et al., 2010). In contrast, the precursor histidine, which confers the dipeptide's bioactive properties, is one of the essential amino acids found in sufficient quantities in the serum. Carnosine is the only histidine-containing dipeptide found in human tissues. Studies reported gender-based differences in the concentrations of carnosine found in the muscle, potentially linked to androgen levels (Boldyrev et al., 2013), while other authors did not find any difference (Stuerenburg and Kunze, 1999).
Upon ageing, the degenerative loss of skeletal muscle mass, also known as sarcopenia, is concomitant with a decline in carnosine concentrations, resulting in decreased muscle power and strength, and increased frequency of muscle fatigue (Rizvi and Jha, 2011). Tallon, et al. (2007) compared the carnosine concentrations in biopsy samples of the vastus lateralis of elderly patients - who underwent knee arthroplasty due to osteoarthritis - to those of young, relatively active subjects. A 53\% reduction in carnosine concentrations was found in the type II muscle fibers (or fast-twitch muscle fibers) of the elderly subjects, while carnosine concentrations in type I muscle fibers (slow-twitch) did not differ from those measured in the young subjects. The age-related reduction of carnosine concentrations in the muscle has been widely documented (Boldyrev et al., 2013; Stuerenburg and Kunze 1999) and supplementation with $\beta$-alanine was shown to be beneficial in elderly patients as evidenced by an increase in carnosine concentrations and muscle exercise capacity (Derave et al., 2007).

\section{Heath benefits}

Carnosine and its precursor $\beta$-alanine both exhibit a robust list of health benefits including antioxidant activity, metal chelating, interaction with lipid peroxidation end products, anti-glycation activity, prevention of protein crosslinking, carbonyl group scavenging, reduction of diabetic pathologies, anti-inflammatory activity, and prevention of senile cataracts (Bauchart et al., 2007; Kyriazis, 2010). Table 2 summarizes the anti-oxidant, anti-glycation and anti-inflammation activity of carnosine as well as the health performance associated with the intake of carnosine.

The anti-oxidant properties of carnosine stem from its ability to directly interact with singlet oxygen, as well as its ability to scavenge peroxyl and superoxide radicals, thereby preventing the negative effects resulting from the production of reactive oxygen species (Preedy, 2015). The direct interaction of carnosine with these reactive species prevents the production of lipid peroxidation end products such as malondialdehyde (MDA), which are deleterious to an organism. Another aspect of the anti-oxidant activity of carnosine resides in its ability to chelate metals, including transition metals, which would otherwise give rise to free radicals via the Fenton reaction. Carnosine was also documented for its ability to prevent copper- and zinc-associated neurotoxicity by binding directly with divalent metals (Derave et al. 2010). In a study conducted by Evran et al. (2014), a carnosine pretreatment in rats led to decreased lactate dehydrogenase and aspartate aminotransferase in plasma, reflecting its role during oxidative stress while improved histopathological changes were observed in heart tissues. The same protective effect was confirmed by Kalaz et al. (2014). Upon ageing, carnosine levels were found to decrease in rats hence resulting in an increased susceptibility toward ageing-related oxidative stress. In a D-galactose-induced liver damage model, the authors showed that supplementation with carnosine was able to maintain the pro-oxidant status found in the liver together with histopathological ameliorations (Kalaz et al., 2014). Similarly, carnosine has cardiovascular protective properties reliant on its ability to inhibit low-density lipoprotein oxidation (Park et al., 2005). The anti-proliferative properties of carnosine were also investigated in colon cancer cells where canosine was shown to reduce the generation of reactive oxygen species (ROS) (Iovine et al., 2012).

Glycation is the process by which aldehydes and ketones react with proteins to form aberrant glycated proteins. The abnormal proteins proceed to crosslinking into larger complexes of dysfunctional proteins and the accumulation of such cross-linked glycated 
proteins subsequently leads to the formation of advanced glycation end products (AGEs). AGEs react in turn with reactive oxygen species, which leads to the development of age-related chronic degenerative diseases (Kyriazis, 2010). When proteins undergo glycation or oxidation, damage occurs in the amino acid side groups, during which carbonyl groups are being formed. These carbonyl groups cause the target proteins to become aberrant, crosslink with other proteins and turn into AGEs (Brownson and Hipkiss, 2000). Glycation and oxidation are inherent aspects of ageing leading to age-related accumulation of AGEs in tissues (Brownson and Hipkiss, 2000). Individuals suffering from diabetes have a greater prevalence of glycation events since there is more sugar sources available in their body. These glycation reactions serve as the initial step in the development of diabetic pathologies (Hipkiss et al., 1995). In a study by Abdelkader et al. (2016), carnosine was shown to have effective anti-glycation activity in cultured porcine lenses and human lens epithelial cell models. Even in the absence of a diabetic condition, the use of carnosine as a supplement may also help reduce glycosylated damage on lens redox glutathione system. Further investigations are warranted on the pharmacology of carnosine as an inhibitory agent against the development of glycation-induced diabetic cataract and other diseases (Babizhayev, 2012; Peters et al., 2014). For example, hypertension is a large concern for diabetic patients and arises from the sugar-mediated crosslinking of proteins in blood vessels, which causes a reduction in the elasticity of the vessel (Hipkiss et al., 2001; Yapislar and Taskin, 2014). Carnosine interferes with glycation at different stages of the process. First, carnosine can bind directly with small ketones and aldehydes to form non-mutagenic glycated carnosine (Brownson and Hipkiss, 2000, Hipkiss et al., 1995), functioning as a sacrificial agent in these reactions (Hipkiss et al., 2001). Such a process will in turn protect other proteins from being glycated and becoming dysfunctional. One of the most striking properties of carnosine resides in its carbonyl group scavenging activity. Carnosine is capable of scavenging and interacting directly with carbonyl groups on proteins to form protein-carbonyl-carnosine adducts through carnosinylation, which in turn inhibits crosslinking events and AGE formation of aberrant proteins (Brownson and Hipkiss, 2000). Interestingly, carnosine is also able to bind low molecular weight AGEs (Brownson and Hipkiss, 2000). The final fate of these protein-carbonyl-carnosine adducts has not yet been established, however, current literature proposed three potential outcomes: (1) these adducts remain in the cell as lipofuscin, (2) they exit the cell via exocytosis, or (3) they undergo proteasomal and lysosomal breakdown (Hipkiss et al., 2001). As an anti-glycation agent, there is strong supportive evidence that carnosine indeed delays the effects of ageing at a cellular level. Highly oxidized proteins resulting from age-associated oxidation are known to induce senescence, hence reducing the maximum division potential of cells and resistance to proteolytic degradation (Hipkiss et al., 2001). Reliant on its anti-oxidant and anti-glycation activity, carnosine may prove to be a valuable bioactive component in the inhibition of certain ageing processes.

Son et al. (2008) examined the anti-inflammatory effects of carnosine in the human intestinal epithelial cell line Caco-2 as measured by interleukin (IL)- 8 secretion. Pretreatment with carnosine was found to decrease the level of IL- 8 secretion in hydrogen peroxide $\left(\mathrm{H}_{2} \mathrm{O}_{2}\right)$ - and tumor necrosis factor (TNF)- $\alpha$-stimulated Caco- 2 cells. A $67 \%$ and $89 \%$ reduction in IL- 8 secretion was observed in Caco-2 cells treated with $5 \mathrm{mM}$ and $50 \mathrm{mM}$, respectively. Carnosine concentrations of up to $50 \mathrm{mM}$ did not exhibit any cytotoxic effects in this cell model. Interestingly, the carnosinemediated inhibition of IL-8 secretion occurred through a translational, rather than a transcriptional mechanism, and required the presence of the dipeptide in its intact form. Carnosine was found to inhibit the phosphorylation of the translation initiation factor eIF4E, which has for role to prevent any further translational mechanisms. This departs from the anti-inflammatory mechanisms of other histidine-containing dipeptides (HCD) such as glycinehistidine (Gly-His), alanine-histidine (Ala-His), and the methylated form of carnosine, anserine ( $\beta$-Ala-1-methyl-His), which inhibited both the mRNA expression and secretion of IL-8. One study explored the effect of carnosine supplementation in elderly people presenting verbal episodic memory and reported decreased secretion of inflammatory cytokines, such as monocyte chemoattractant protein (MCP)-1 and IL-8. A treatment with carnosine was shown to preserve delayed recall and maintain brain perfusion in the elderly subjects (Hisatsune et al., 2016). In a study by Sun et al. (2017), the therapeutic potential of carnosine was investigated in a sepsis-induced male albino rats. The administration of carnosine was shown to renormalize the activity of anti-oxidative enzymes (i.e., catalase, superoxide dismutase, glutathione peroxidase and myeloperoxidase activities) and simultaneously decrease the secretion of pro-inflammatory cytokines (i.e., IL- $\beta$, TNF- $\alpha$, and IL8 ). Histopathological analysis confirmed that the tissue damages associated with sepsis were significantly reduced upon carnosine treatment, in association with the upregulation of $\operatorname{I\kappa B} \alpha$ and the down-regulation of p65 and p-IKK $\alpha / \beta$ (Ser 180/Ser 181).

Carnosine, in its acetylated form, i.e., $\mathrm{N}$-alpha-acetyl-carnosine (NAC), was reported to prevent crosslinking events occurring between the age-related glycated crystalline molecules present in the lens of the eye, an event leading to senile cataracts. A number of studies also suggested that NAC may reduce telomere shortening, which is another characteristic feature associated with ageing (Kyriazis, 2010). NAC is currently marketed as a liquid ophthalmic solution to be used topically on the eye and is reported to improve visual acuity and glare sensitivity, however these claims are not supported by organizations such as the Royal College of Ophthalmalogists in the U.K. (Kyriazis, 2010). In a study by Ding et al., (2018), the oral ingestion of carnosine in elderly people possessing the APOE e4 allele, a known risk gene for the development of $\mathrm{AD}$, led to a reduced decline in their progressive memory, hence inhibiting the development of AD in APOE e4+ individuals.

Upon oral administration of carnosine in a mouse model of permanent focal cerebral ischemia, infarct size and neuronal damage were significantly decreased together with reduced levels of reactive oxygen species in the ischemic brain (Rajanikant et al., 2007). Similarly, the neuroprotective properties of carnosine were also studied for their compensatory effect on brain damage induced by ischemic injury. The robust anti-oxidant, anti-excitotoxic, and mitochondria protecting activities of carnosine mainly rely on its protective effects against oxygen-glucose deprivation and against accumulation of reactive oxygen species (Bae et al., 2013). In a mice model of salsolinol-induced neurotoxicity, carnosine was shown to decrease the MDA and restore the activity of Glutathione(GSH), Superoxide dismutase (SOD) and catalase along with the reduced apoptosis and Reactive Oxygen Species (ROS) production, suggesting its therapeutic effect in salsolinol-induced Parkinson's disease (PD) (Zhao et al., 2017). In summary, an increasing number of studies have provided supportive evidence on the capacity of carnosine to restore or promote the activity of antioxidant enzymes, its ability to inhibit lipid oxidation together with its capacity to inhibit anti-crosslinking between amyloid- $\beta$ and prion peptides, which altogether encourage the use of carnosine as a preventive and therapeutic approach against $\mathrm{PD}$ and $\mathrm{AD}$.

The ingestion of carnosine was also determined to exert a therapeutic effect on chronic metabolic disorders, a property which is likely associated with its potent anti-oxidative, anti-glycation, 
Table 2. Biological activity of carnosine defined by in vitro and in vivo studies

\begin{tabular}{|c|c|c|c|}
\hline Bioactive activity & Experimental Model & Observed effects & Reference \\
\hline \multirow[t]{13}{*}{ Anti-oxidant } & Chemical detection & $\begin{array}{l}\text { Inactivate free radicals; Metal-chelating, such } \\
\text { as copper;Conjugating with potentially toxic } \\
\text { aldehydic lipid oxidation products }\end{array}$ & Decker et al., 2000 \\
\hline & Cerebellum granule cells & $\begin{array}{l}\downarrow \text { ROS signal; } \downarrow \text { Excitotoxic effect of } \\
\text { N-methyl-d-aspartic acid (NMDA) }\end{array}$ & Boldyrev et al., 2004 \\
\hline & Rat & $\downarrow$ Lactate dehydrogenase & Park et al., 2005 \\
\hline & $\begin{array}{l}\text { Wistar rats and } \\
\text { Mongolian gerbils }\end{array}$ & $\begin{array}{l}\downarrow \text { Lipid peroxidation of brain membrane; } \\
\uparrow \text { Resistance of neuronal membranes under } \\
\text { oxidation; } \downarrow \text { malonyl dialdehyde (MDA) }\end{array}$ & Dobrota et al., 2005 \\
\hline & Chemical detection & Metal-chelating; $\downarrow$ Lipid peroxidation & Derave et al., 2010 \\
\hline & Colon cancer cells & $\downarrow R O S ; \downarrow$ ERK1/2 phosphorylation; $\downarrow$ Proliferation & lovine et al., 2012 \\
\hline & Sprague-Dawley male rats & $\begin{array}{l}\downarrow \text { Oxidative stress; Restores the histopathological } \\
\text { and biochemical signs; } \downarrow \text { Apoptotic condition }\end{array}$ & Aydin et al., 2015 \\
\hline & Mice & $\begin{array}{l}\text { } \text { Glutathione peroxidase (GPX), Superoxide dismutase } \\
\text { (SOD), and Catalase; } \downarrow \text { MDA ; } \downarrow \text { Reactive oxygen species }\end{array}$ & Tsai et al., 2010 \\
\hline & $\begin{array}{l}\text { Recreationally-active } \\
\text { men volunteer }\end{array}$ & 个GSH 个CAT, PRX2, SOD1, and TRX1 & $\begin{array}{l}\text { Varanoske et } \\
\text { al., } 2017\end{array}$ \\
\hline & Broiler chickens & 个Antioxidant enzymes; $\downarrow$ MDA and carbonyl compounds & Cong et al., 2017 \\
\hline & Wistar rats & 个CAT,SOD and total antioxidant capacity (TAC) & $\begin{array}{l}\text { Hasanein and } \\
\text { Felegari, } 2017\end{array}$ \\
\hline & Cirrhotic rats & $\begin{array}{l}\uparrow \text { Locomotor activity; } \downarrow \text { ROS formation; } \downarrow \text { Liver } \\
\text { TBARS; } \uparrow \text { GSH in liver; } \downarrow \text { Protein carbonylation; } \\
\downarrow \text { Liver fibrogenesis; } \downarrow \text { Liverfailure }\end{array}$ & $\begin{array}{l}\text { Jamshidzadeh } \\
\text { et al., } 2017\end{array}$ \\
\hline & $\begin{array}{l}\text { Human erythrocytes } \\
\text { and lymphocytes }\end{array}$ & $\begin{array}{l}\downarrow \text { Oxidative damage; Restores enzyme } \\
\text { activities and antioxidant power }\end{array}$ & $\begin{array}{l}\text { Husain and } \\
\text { Mahmood, } 2017\end{array}$ \\
\hline \multirow[t]{6}{*}{ Anti-glycation } & Chemical detection & $\begin{array}{l}\uparrow \text { Glyc3P-induced loss of enzyme } \\
\text { activity; } \downarrow \text { Protein modification }\end{array}$ & Seidler, 2000 \\
\hline & $\begin{array}{l}\text { Human Peritoneal } \\
\text { Mesothelial Cells (HPMC) }\end{array}$ & $\begin{array}{l}\text { 个HPMC viability; } \downarrow \text { Advanced glycation end products (AGE) } \\
\text { complications; Protection cellular protein from modification; }\end{array}$ & $\begin{array}{l}\text { Alhamdani et } \\
\text { al., } 2007\end{array}$ \\
\hline & E. coli K-12 Strains & $\uparrow$ Cell viability; $\downarrow$ Glycation; $\downarrow$ Methylglyoxal toxicity & Pepper et al., 2010 \\
\hline & Gray chinchilla rabbits & $\downarrow$ Gycation-induced diabetic cataract & $\begin{array}{l}\text { Babizhayev et } \\
\text { al., } 2012\end{array}$ \\
\hline & $\begin{array}{l}\text { Human lens epithelial } \\
\text { cell models }\end{array}$ & $\downarrow$ Glycosylated damage; $\uparrow$ Lens cell viability & $\begin{array}{l}\text { Abdelkader et } \\
\text { al., } 2016\end{array}$ \\
\hline & Aged rats & $\begin{array}{l}\downarrow A G E \text { and MDA; } \downarrow \text { Protein carbonyl and } \\
\text { advanced oxidized protein products }\end{array}$ & Bingül et al., 2017 \\
\hline \multirow[t]{8}{*}{ Anti-inflammatory } & Caco- 2 cells & $\downarrow$ IL-8 secretion & Son et al., 2008 \\
\hline & Male Balb/cA mice & $\downarrow$ c-reactive protein (CRP), IL-6 tumor and TNF- $\alpha$ & Liu et al., 2008 \\
\hline & Balb/cA mice & $\downarrow$ IL-6, TNF- $\alpha$, and MCP-1 & Yan et al., 2009 \\
\hline & Mice & $\downarrow$ TNF- $\alpha$ and IL- 6 levels & Tsai et al., 2010 \\
\hline & Elderly people & $\downarrow M C P-1$ and IL-8 & $\begin{array}{l}\text { Hisatsune et } \\
\text { al., } 2016\end{array}$ \\
\hline & $\begin{array}{l}\text { Sepsis-induced } \\
\text { male albino rats. }\end{array}$ & $\begin{array}{l}\downarrow I L-\beta, \text { TNF- } \alpha \text {, and IL-8; } \uparrow \text { IKB } \alpha ; \downarrow \text { p65 and } \\
\text { p-IKK } \alpha / \beta \text { (Ser 180/Ser 181); } \downarrow \text { Lung Injury }\end{array}$ & Sun et al., 2017 \\
\hline & $\begin{array}{l}\text { Human cancer cell } \\
\text { line HepG2 }\end{array}$ & $\downarrow P I 3 K$ and Akt ; 个Caspase- $8 ; \downarrow$ Proliferation; 个apoptosis & Liu et al., 2017 \\
\hline & Wistar rats & $\downarrow$ TNF- $\alpha$ and IL-6 levels & $\begin{array}{l}\text { Hasanein and } \\
\text { Felegari, } 2017\end{array}$ \\
\hline
\end{tabular}


and anti-inflammatory properties. Houjeghani et al. (2018) investigated the effect of carnosine supplementation on the clinical symptoms of type 2 diabetes patients over a 12 week-period. Fat mass and fat-free mass were significantly lower in the intervention group together with lower levels of fasting blood glucose, glycated hemoglobin, carboxymethyl lysine, serum triglyceride and TNF- $\alpha$, compared to the placebo group. Supplementation with carnosine also led to lower levels of hemoglobin A1c, urinary albumin excretion, tubular damage marker $\alpha 1$-microglobulin (A1M), and MDA while higher antioxidant capacity was observed. Altogether, these phenomena are known to promote glycemic control and renal function, particularly in pediatric patients suffering from diabetic nephropathy (Elbarbary et al., 2018). In a recent pilot randomised controlled trial, fasting insulin and insulin resistance was hampered in individuals who were given carnosine (de Courten et al., 2016). The properties of carnosine were also examined in obese individuals with the aim to investigate its effect on the composition of plasma lipidome (Baye et al., 2017). As expected, carnosine supplementation led to lower levels of trihexosylceramide (THC) and free cholesterol, whereas the concentrations of phosphatidylcholine (PC) were higher. From the perspective of lipid metabolism, the beneficial roles of carnosine may be associated with regulation of signal transmission, cell adhesion, growth factor and protein transporters, which further contribute to regulation of insulin resistance and glucose uptake.

In a study by Derave et al. (2007), the anti-fatigue activity of carnosine was evaluated in a cohort of well-trained, 400-m sprint runners upon oral supplementation with $\beta$-alanine for 4 weeks. As expected, the oral intake of $\beta$-alanine was shown to increase carnosine content in both the soleus $(+47 \%)$ and the gastrocnemius $(+37 \%)$. Compared with the placebo group, the dynamic knee extension torque was improved significantly; this was the first report that, under repeated exhaustive contractions, muscle fatigue may be attenuated in the later stages of exercise (bouts 4 and 5) under the effect of carnosine. Similar results were obtained in a young cohort of sportive individuals, whereby carnosine intramuscular contents were found to be higher together with fatigue attenuation (Varanoske et al., 2017). In the elderly population, a reduction in muscular endurance was shown to be associated with a significant decline in skeletal muscle carnosine concentrations (Tallon et al., 2007). Upon supplementation with $\beta$-alanine, higher carnosine levels were detected in the elderly subjects ( $>55$ years) along with improvements in the time-to-exhaustion test, while there were no changes in the placebo treatment (del Favero et al., 2012). In a mouse model, the administration of carnosine $(1.8 \%)$ resulted in markedly higher carnosine levels $(+160 \%)$ together with increased resistance to fatigue in the soleus $(+2-4 \%)$ and a marked leftward shift of the force-frequency relation under the $+10-31 \%$ relative forces in the extensor digitorum longus as well as in the soleus (Everaert et al., 2013). In a study by Dutka et al.(2011), carnosine was reported to improve $\mathrm{Ca}^{2+}$ sensitivity in contractile apparatus and promote $\mathrm{Ca}^{2+}$ release in the sarcoplasmic reticulum of human skeletal muscle fibers. A significant biological property of carnosine is its buffering capacity to whereby higher levels of carnosine enhance the buffering capacity within the muscle hence delaying muscle fatigue (Zanella et al., 2017). Oral supplementation with $\beta$-alanine elevates endogenous level of carnosine leading to suppression of muscular fatigue. This has encouraged the public to regard $\beta$-alanine as a powerful supplement especially among athletes and elderly people today.

Current research on its ability to reduce oxidative stress, buffer $\mathrm{pH}$ microenvironment and prevent age-related accumulation of glycation products provides convincing evidence that carnosine is a highly beneficial bioactive compound. In brief, the potential thera- peutic effect of carnosine on chronic metabolic disorders mainly relies on its excellent anti-oxidative, anti-glycation and anti-inflammatory activities, as well as its role in the regulation of anti-oxidant enzymes, pro-inflammatory cytokines and I $\kappa \mathrm{B} \alpha$ expression, etc. Further investigations on its physiology and on its associated genetics will be required to gain a fuller understanding of the varied functions and health-related applications of this intriguing molecule.

\section{Safety considerations}

Carnosine is a safe bioactive component in its dietary and supplemental forms. There have been no significant side effects documented, likely owing to the fact humans have abundant levels of serum carnosinase which serve to rapidly degrade carnosine in the body. The only side effect that has been reported is paresthesia, which is a benign sensation of tingling and numbness, when circulating $\beta$-alanine concentrations exceed $100 \mu \mathrm{M}$, compared with less than $0.5 \mu \mathrm{M}$ of $\beta$-alanine concentration (Derave et al., 2010). To prevent this undesirable side effect, it is recommended that individuals take a slow-release $\beta$-alanine supplement (Boldyrev et al., 2013) or not exceed a dose of $10 \mathrm{mg}$ per kilogram of bodyweight (Derave et al., 2010). In a human clinical trial assessing the health benefit of carnosine, carnosine doses ranging from 0.8 to $2.8 \mathrm{~g} /$ day were used upon oral administration while the $\beta$-alanine dosing regimens always ranged from 1.6 to $6.4 \mathrm{~g}$ /day, which all showed to be good tolerance and no side effect was checked in the previous study (Chez et al., 2002; Elbarbary et al., 2018; Houjeghani et al., 2018). Despite significant differences in baseline values, supplementation with carnosine or $\beta$-alanine showed similar increases in the gastrocnemius and the soleus muscles regardless of gender. The congenital lack of the degradative enzyme carnosinase results in serious conditions such as carnosinemia (Kyriazis, 2010). Hence consumers have expressed concern that too large a dose of carnosine may lead to overwhelming concentrations of carnosine resulting in carnosinemia. This was, however, shown not to occur in normal individuals with abundant levels of serum carnosinase.

Overall, carnosine and its precursor $\beta$-alanine are safe bioactive food components have tremendous health-beneficial properties, which heavily outweigh the sole side effect ever reported, i.e., paresthesia.

\section{Conclusion}

Carnosine is a naturally occurring dipeptide, which may be ingested and absorbed via consumption of meat or dietary supplements. A growing body of evidence supports its role as a potent antioxidant and anti-glycation bioactive dietary dipeptide along with studies strongly supporting its bioaccessibility, bioavailability, and health benefits. Due to its tightly regulated metabolism managed by the concerted activities of carnosine synthetase and carnosinase, carnosine proves to be a safe food component as part of a balanced dietary intake of meat-derived proteins. Given the known properties of carnosine as well as the age-related reduction in muscle carnosine levels, supplementation with carnosine or $\beta$-alanine should be considered as an efficient approach to improve the endurance of muscle. Despite an impressive body of carnosinefocused studies, research gaps remain. Human cohort studies are lacking to determine the actual bioavailability of carnosine and how this may impact on health performance. Further investigations are warranted on the mechanisms underlying carnosine-mediated increased health performance, such as cell signaling pathways, 
impact on the intestinal microflora and overall gut health, and genome.

\section{References}

Abe, H. (2000). Role of histidine-related compounds as intracellular proton buffering constituents in vertebrate muscle. Biochemistry Biokhimiia. 65(7): 757-765.

Abdelkader, H., Longman, M., Alany, R.G., and Pierscionek, B. (2016). On the anticataractogenic effects of I-carnosine: is it best described as an antioxidant, metal-chelating agent or glycation inhibitor. Oxid. Med. Cell. Longevity 3: 1-11.

Alhamdani, M.-S.S., Abdul-Hameed, A.-M., Abbas, F.K., Jaleel, N.A., and AlTaee, M.F. (2007). Antiglycation and antioxidant effect of carnosine against glucose degradation products in peritoneal mesothelial cells. Nephron Clin. Pract. 107(1): 26-34.

Aydin, S., Ogeturk, M., Kuloglu, T., Kavakli, A., and Aydin, S. (2015). Effect of carnosine supplementation on apoptosis and irisin, total oxidant and antioxidants levels in the serum, liver and lung tissues in rats exposed to formaldehyde inhalation. Peptides. 64: 14-23.

Babizhayev, M.A. (2012). Bioactivation antioxidant and transglycating properties of n-acetylcarnosine autoinduction prodrug of a dipeptide l-carnosine in mucoadhesive drug delivery eye-drop formulation: powerful eye health application technique and therapeutic platform. Drug Test. Anal. 4(6): 468-485.

Bae, O.-N., Serfozo, K., Baek, S.-H., Lee, K.Y., Dorrance, A., Rumbeiha, W., Fitzgerald, S.D., Farooq, M.U., Naravelta, B., and Bhatt, A. (2013). Safety and efficacy evaluation of carnosine, an endogenous neuroprotective agent for ischemic stroke. Stroke. 44(1): 205-212.

Bauchart, C., Savaryauzeloux, I., Patureau, M.P., Thomas, E., Morzel, M. and Rémond, D. (2007). Carnosine concentration of ingested meat affects carnosine net release into the portal vein of minipigs. J. Nutri. 137(3): 589-593.

Bhardwaj, R.K., Herrera-Ruiz, D., Eltoukhy, N., Saad, M., and Knipp, G.T. (2006). The functional evaluation of human peptide/histidine transporter 1 (hPHT1) in transiently transfected COS-7 cells. Eur. J. Pharm. Sci. 27(5): 533-542.

Boldyrev, A., Bulygina, E., Leinsoo, T., Petrushanko, I., Tsubone, S., and Abe, H. (2004). Protection of neuronal cells against reactive oxygen species by carnosine and related compounds. Comp. Biochem. Physiol. B Biochem. Mol. Biol. 137(1): 81-88.

Baye, E., Ukropec, J., de Courten, M.P., Vallova, S., Krumpolec, P., Kurdiova, T., Aldini, G., Ukropcova, B., and de Courten, B. (2017). Effect of carnosine supplementation on the plasma lipidome in overweight and obese adults: a pilot randomised controlled trial. Sci. Rep. 7(1): 17458-17463.

Bingül, İ., Yılmaz, Z., Aydın, A.F., Çoban, J., Doğru-Abbasoğlu, S., and Uysal, M. (2017). Antiglycation and anti-oxidant efficiency of carnosine in the plasma and liver of aged rats. Geriatr. Gerontol. Int. 17(12): 2610-2614.

Boldyrev, A.A., Aldini, G., and Derave, W. (2013). Physiology and pathophysiology of carnosine. Physiol Rev. 93(4): 1803-1845.

Brownson, C., and Hipkiss, A.R. (2000). Carnosine reacts with a glycated protein. Free Radical Bio. Med. 28(10): 1564-1570.

Chan, K., De Cker, E., and Means, W. (1993). Extraction and activity of carnosine, a naturally occurring antioxidant in beef muscle. J. Food Sci. 58(1): 1-4.

Chez, M.G., Buchanan, C.P., Aimonovitch, M.C., Becker, M., Schaefer, K., Black, C., and Komen, J. (2002). Double-blind, placebo-controlled study of I-carnosine supplementation in children with autistic spectrum disorders. J. Child Neurol. 17(11): 833-840.

Cong, J., Zhang, L., Li, J., Wang, S., Gao, F., and Zhou, G. (2017). Effects of dietary supplementation with carnosine on growth performance, meat quality, antioxidant capacity and muscle fiber characteristics in broiler chickens. J. Sci. Food Agr. 97(11): 3733-3741.

Crush, K. (1970). Carnosine and related substances in animal tissues. Comp. Biochem. Phys. 34(1): 3-30.

de Courten, B., Jakubova, M., de Courten, M.P., Kukurova, I.J., Vallova, S., Krumpolec, P., Valkovic, L., Kurdiova, T., Garzon, D., and Barbaresi,
S. (2016). Effects of carnosine supplementation on glucose metabolism: Pilot clinical trial. Obesity. 24(5): 1027-1034.

Decker, E., Livisay, S., and Zhou, S. (2000). A re-evaluation of the antioxidant activity of purified carnosine. Biochem. Biokhimiia. 65(7): 766-770.

del Favero, S., Roschel, H., Solis, M.Y., Hayashi, A.P., Artioli, G.G., Otaduy, M.C., Benatti, F.B., Harris, R.C., Wise, J.A., and Leite, C.C. (2012). Beta-alanine $\left(\right.$ Carnosyn $\left.^{\mathrm{TM}}\right)$ supplementation in elderly subjects $(60-80$ years): effects on muscle carnosine content and physical capacity. Amino Acids. 43(1): 49-56.

Derave, W., Everaert, I., Beeckman, S., and Baguet, A. (2010). Muscle carnosine metabolism and $\beta$-alanine supplementation in relation to exercise and training. Sports Med. 40(3): 247-263.

Derave, W., Ozdemir, M.S., Harris, R.C., Pottier, A., Reyngoudt, H., Koppo, K., Wise, J.A., and Achten, E. (2007). $\beta$-Alanine supplementation augments muscle carnosine content and attenuates fatigue during repeated isokinetic contraction bouts in trained sprinters. J. Appl. Physiol. 103(5): 1736-1743.

di Pierro, F., Bertuccioli, A., Bressan, A., and Rapacioli, G. (2011). Carnosine-based supplement. Nutrafoods 10(2-3): 43-47.

Ding, Q., Tanigawa, K., Kaneko, J., Totsuka, M., Katakura, Y., Imabayashi, E., Matsuda, H., and Hisatsune, T. (2018). Anserine/carnosine supplementation preserves blood flow in the prefrontal brain of elderly people carrying APOE e4. Aging Dis. 9(3): 334-342.

Dobrota, D., Fedorova, T., Stvolinsky, S., Babusikova, E., Likavcanova, K., Drgova, A., Strapkova, A., and Boldyrev, A. (2005). Carnosine protects the brain of rats and Mongolian gerbils against ischemic injury: afterstroke-effect. Neurochem. Res. 30(10): 1283-1288.

Dutka, T.L., Lamboley, C.R., McKenna, M.J., Murphy, R.M., and Lamb, G.D. (2011). Effects of carnosine on contractile apparatus $\mathrm{Ca}^{2+}$ sensitivity and sarcoplasmic reticulum $\mathrm{Ca}^{2+}$ release in human skeletal muscle fibers. J. Appl. Physiol. 112(5): 728-736.

Elbarbary, N.S., Ismail, E.A.R., El-Naggar, A.R., Hamouda, M.H., and ElHamamsy, M. (2018). The effect of 12 weeks carnosine supplementation on renal functional integrity and oxidative stress in pediatric patients with diabetic nephropathy: a randomized placebo-controlled trial. Pediatr. Diabetes. 19(3): 470-477.

Everaert, I., Stegen, S., Vanheel, B., Taes, Y., and Derave, W. (2013). Effect of beta-alanine and carnosine supplementation on muscle contractility in mice. Med. Sci. Sport Exer. 45(1): 43-51.

Evran, B., Karpuzoğlu, H., Develi, S., Kalaz, E.B., Soluk-Tekkeşin, M., Olgaç, V., Doğru-Abbasoğlu, S., and Uysal, M. (2014). Effects of carnosine on prooxidant-antioxidant status in heart tissue, plasma and erythrocytes of rats with isoproterenol-induced myocardial infarction. Pharmacol. Rep. 66(1): 81-86.

Ferraris, R.P., Diamond, J., and Kwan, W.W. (1988). Dietary regulation of intestinal transport of the dipeptide carnosine. Am. J. Physiol-Gastr. Liver Physiol. 255(2): 143-150.

Gil-Agustí, M., Esteve-Romero, J., and Carda-Broch, S. (2008). Anserine and carnosine determination in meat samples by pure micellar liquid chromatography. J. Chromatogr. A. 1189(1-2): 444-450.

Hasanein, P., and Felegari, Z. (2017). Chelating effects of carnosine in ameliorating nickel-induced nephrotoxicity in rats. Can. J. Physiol. Pharm. 95(12): 1426-1432.

Hill, C., Harris, R.C., Kim, H., Harris, B., Sale, C., Boobis, L., Kim, C., and Wise, J.A. (2007). Influence of $\beta$-alanine supplementation on skeleta muscle carnosine concentrations and high intensity cycling capacity. Amino Acids. 32(2): 225-233.

Hipkiss, A.R., Michaelis, J., and Syrris, P. (1995). Non-enzymatic glycosylation of the dipeptide $L$ carnosine, a potential anti-protein-cross-linking agent. FEBS Letters. 371(1): 81-85.

Hipkiss, A.R., Brownson, C., and Carrier, M.J. (2001). Carnosine, the anti-ageing, anti-oxidant dipeptide, may react with protein carbonyl groups. Mech. Ageing Dev. 122(13): 1431-1445.

Hisatsune, T., Kaneko, J., Kurashige, H., Cao, Y., Satsu, H., Totsuka, M., Katakura, Y., Imabayashi, E., and Matsuda, H. (2016). Effect of anserine/ carnosine supplementation on verbal episodic memory in elderly people. J. Alzheimer's Disease. 50(1): 149-159.

Houjeghani, S., Kheirouri, S., Faraji, E., and Jafarabadi, M.A. (2018). ICarnosine supplementation attenuated fasting glucose, triglycerides, advanced glycation end products, and tumor necrosis factor- $\alpha$ levels 
in patients with type 2 diabetes: a double-blind placebo-controlled randomized clinical trial. Nutr. Res. 49: 96-106.

Huang, S.-C., and Kuo, J. (2000). Concentrations and antioxidative activity of anserine and carnosine in poultry meat extracts treated with demineralization and papain. Proc. Natl. Sci. Counc., Life Sci. 24(4): 193-201.

Husain, N., and Mahmood, R. (2017). Copper (II)-induced Cytotoxicity and Oxidative Stress in Human Blood Cells and its Attenuation by Carnosine. Free Radical Bio. Med. 108: 21-28.

lovine, B., lannella, M.L., Nocella, F., Pricolo, M.R., and Bevilacqua, M.A. (2012). Carnosine inhibits KRAS-mediated HCT116 proliferation by affecting ATP and ROS production. Cancer Lett. 315(2): 122-128.

Jamshidzadeh, A., Heidari, R., Latifpour, Z., Ommati, M.M., Abdoli, N., Mousavi, S., Azarpira, N., Zarei, A., Zarei, M., and Asadi, B. (2017) Carnosine ameliorates liver fibrosis and hyperammonemia in cirrhotic rats. Clin. Res. Hepatol. Gas. 41(4): 424-434.

Jappar, D., Hu, Y., Keep, R.F., and Smith, D.E. (2009). Transport mechanisms of carnosine in SKPT cells: contribution of apical and basolateral membrane transporters. Pharm. Res. 6(1): 172-181.

Jones, G., Smith, M., and Harris, R. (2011). Imidazole dipeptide content of dietary sources commonly consumed within the British diet. Pro. Nutr. Soc.70: 363-372.

Jung, S., Bae, Y.S., Kim, H.J., Jayasena, D.D., Lee, J.H., Park, H.B., Heo, K.N., and Jo, C. (2013). Carnosine, anserine, creatine, and inosine 5 '-monophosphate contents in breast and thigh meats from 5 lines of Korean native chicken. Poultry Sci. 92(12): 3275-3282.

Kalaz, E.B., Çoban, J., Aydın, A.F., Doğan-Ekici, I., Doğru-Abbasoğlu, S., Öztezcan, S., and Uysal, M. (2014). Carnosine and taurine treatments decreased oxidative stress and tissue damage induced by D-galactose in rat liver. J. Physiol. Biochem. 70(1): 15-25.

Kantha, S.S., Takeuchi, M., Watabe, S., and Ochi, H. (2000). HPLC determination of carnosine in commercial canned soups and natural meat extracts. LWT-Food Sci. Technol. 33(1): 60-62.

Kyriazis, M. (2010). Anti-ageing potential of carnosine: approaches toward successful ageing. Drug Discovery Today: Ther. Strategies 7(3-4): 45-49.

Lee, H.J., Jayasena, D.D., Kim, S.H., Kim, H.J., Heo, K.N., Song, J.E., and Jo, C. (2015). Comparison of bioactive compounds and quality traits of breast meat from Korean native ducks and commercial ducks. Korean J. Food Sci. An. 35(1): 114-120.

Lenney, J.F., Peppers, S.C., Kucera-Orallo, C.M., and George, R. (1985). Characterization of human tissue carnosinase. Biochem. J. 228(3): 653-660.

Liu, Q., Peng, P.Y., Dai, J.S., and Xiao, B. (2017). Study in L-carnosine on the proliferation and apoptosis of human hepatocellular carcinoma HepG2 cells. J. Regional Anatomy Operative Surgery. 26(10): 710714.

Liu, W.H., Liu, T.C., and Yin, M.C. (2008). Beneficial effects of histidine and carnosine on ethanol-induced chronic liver injury. Food Chem. Toxicol. 46(5): 1503-1509.

Marcolini, E., Babini, E., Bordoni, A., Di Nunzio, M., Laghi, L., Maczo, A., Picone, G., Szerdahelyi, E., Valli, V., and Capozzi, F. (2015). Bioaccessibility of the bioactive peptide carnosine during in vitro digestion of cured beef meat. J. Agr. Food Chem. 63(20): 4973-4978.

Mora, L., Sentandreu, M.Á., and Toldrá, F. (2008). Contents of creatine, creatinine and carnosine in porcine muscles of different metabolic types. Meat Sci. 79(4): 709-715.

Park, Y.J., Volpe, S.L., and Decker, E.A. (2005). Quantitation of carnosine in humans plasma after dietary consumption of beef. J. Agr. Food Chem. 53(12): 4736-4739.

Peiretti, P.G., Medana, C., Visentin, S., Dal Bello, F., and Meineri, G. (2012) Effect of cooking method on carnosine and its homologues, pentosidine and thiobarbituric acid-reactive substance contents in beef and turkey meat. Food Chem. 132(1): 80-85.

Peiretti, P.G., and Meineri, G. (2015). Carnosine and its homologs in foods. Imidazole Dipeptides Royal Society of Chemistrry, London, UK, 2339.

Pepper, E.D., Farrell, M.J., Nord, G., and Finkel, S.E. (2010). Antiglycation effects of carnosine and other compounds on the long-term survival of Escherichia coli. Appl. Environ. Microb. 76(24): 7925-7930.

Peters, V., Riedl, E., Braunagel, M., Hoger, S., Hauske, S., Pfister, F., Zschockec, J., Lanthalerc, B., Benckb, U., Hammesb, H.P., Krämerb, B.K., Schmitta, C.P., Yard, B.A., and Koppel, H. (2014). Carnosine treatment in combination with ace inhibition in diabetic rats. Regul. Pept. 194 195: 36-40.

Purchas, R.W., Rutherfurd, S.M., Pearce, P.D., Vather, R., and Wilkinson, B.H. (2004). Cooking temperature effects on the forms of iron and levels of several other compounds in beef semitendinosus muscle. Meat Sci. 68(2): 201-207.

Preedy, V.R. (2015). Imidazole dipeptides: chemistry, analysis, function and effects Royal Society of Chemistrry, London, UK, 43-60.

Rajanikant, G., Zemke, D., Senut, M.-C., Frenkel, M.B., Chen, A.F., Gupta, R., and Majid, A. (2007). Carnosine is neuroprotective against permanent focal cerebral ischemia in mice. Stroke. 38(11): 3023-3031.

Rizvi, S.I., and Jha, R. (2011). Strategies for the discovery of anti-aging compounds. Expert Opin. Drug Dis. 6(1): 89-102.

Seidler, N.W. (2000). Carnosine prevents the glycation-induced changes in electrophoretic mobility of aspartate aminotransferase. J. Biochem. Mol.Toxic. 14(4): 215-220.

Son, D.O., Satsu, H., Kiso, Y., and Shimizu, M. (2004). Characterization of carnosine uptake and its physiological function in human intestinal epithelial Caco-2 cells. Biofactors 21(1-4): 395-398.

Son, D.O., Satsu, H., Kiso, Y., Totsuka, M., and Shimizu, M. (2008). Inhibitory effect of carnosine on interleukin- 8 production in intestinal epithelial cells through translational regulation. Cytokine. 42(2): 265-276.

Stuerenburg, H.J., and Kunze, K. (1999). Concentrations of free carnosine (a putative membrane-protective antioxidant) in human muscle biopsies and rat muscles. Arch. Gerontol. Geriat. 29(2): 107-113.

Stvolinsky, S., Bulygina, E., Fedorova, T., Meguro, K., Sato, T., Tyulina, O., Abe, H., and Boldyrev, A. (2010). Biological activity of novel synthetic derivatives of carnosine. Cell Mol. Neurobiol. 30(3): 395-404.

Sun, C., Wu, Q., Zhang, X., He, Q., and Zhao, H. (2017). Mechanistic Evaluation of the Protective Effect of Carnosine on Acute Lung Injury in Sepsis Rats. Pharmacology. 100(5-6): 292-300.

Suzuki, Y., Ito, O., Mukai, N., Takahashi, H., and Takamatsu, K. (2002). High level of skeletal muscle carnosine contributes to the latter half of exercise performance during 30-s maximal cycle ergometer sprinting. JPN J. Physiol. 52(2): 199-205.

Tallon, M.J., Harris, R.C., Maffulli, N., and Tarnopolsky, M.A. (2007). Carnosine, taurine and enzyme activities of human skeletal muscle fibres from elderly subjects with osteoarthritis and young moderately active subjects. Biogerontology. 8(2): 129-137.

Tsai, S.J., Kuo, W.W., Liu, W.H., and Yin, M.-C. (2010). Antioxidative and anti-inflammatory protection from carnosine in the striatum of MPTPtreated mice. J. Agr. Food Chem. 58(21): 11510-11516.

Varanoske, A.N., Hoffman, J.R., Church, D.D., Coker, N.A., Baker, K.M. Dodd, S.J., Oliveira, L.P., Dawson, V.L., Wang, R., and Fukuda, D.H. (2017). $\beta$-Alanine supplementation elevates intramuscular carnosine content and attenuates fatigue in men and women similarly but does not change muscle I-histidine content. Nutr. Res. 48: 16-25.

Varanoske, A.N., Hoffman, J.R., Church, D.D., Jajtner, A.R., Townsend J.R., Beyer, K.S., Oliveira, L.P., Fukuda, D.H., and Stout, J.R. (2017). Effects of Resting Muscle Carnosine Content on the Intramuscular Antioxidant Response to a Bout of Resistance Exercise. Faseb. J. 31: 702-707.

Yan, S.I., Wu, S.t., Yin, M.C., Chen, H.T., and Chen, H.C. (2009). Protective effects from carnosine and histidine on acetaminophen-induced liver injury. J. Food Sci. 74(8): 259-265.

Yapislar, H., and Taskin, E. (2014). L-carnosine alters some hemorheologic and lipid peroxidation parameters in nephrectomized rats. Med. Sci. Monit. 20: 399-405.

Zanella, P.B., Alves, F.D., and de Souza, C.G. (2017). Effects of beta-alanine supplementation on performance and muscle fatigue in athletes and non-athletes of different sports: a systematic review. J. Sports Med. Phy. Fitness. 57: 1132-1141.

Zhao, J., Shi, L., and Zhang, L.R. (2017). Neuroprotective effect of carnosine against salsolinol-induced Parkinson's disease. Exp. Ther. Med. 14(1): 664-670. 